\title{
Dog activity classification with movement sensor placed on the collar
}

\author{
Pekka Kumpulainen $^{1}$, Anna Valldeoriola ${ }^{2}$, Sanni Somppi ${ }^{2}$, Heini Törnqvist ${ }^{2}$, Heli Väätäjä ${ }^{3}$, Päivi \\ Majaranta $^{3}$, Veikko Surakka ${ }^{3}$, Outi Vainio ${ }^{2}$, Miiamaaria V. Kujala ${ }^{2,4}$, Yulia Gizatdinova ${ }^{3}$, Antti \\ Vehkaoja ${ }^{1}$ \\ ${ }^{1}$ Tampere University of \\ Technology \\ Tampere, Finland \\ \{forename.surname\}@tut.fi \\ f Helsinki \\ ${ }^{2}$ University of Helsin
Helsinki, Finland \\ \{forename.surname\}@ \\ helsinki.fi \\ ${ }^{4}$ Aalto University \\ Helsinki, Finland
}

\begin{abstract}
Dog owners are highly motivated in understanding behavior and physiology of their pets and monitoring their wellbeing. Monitoring with a commercially available activity trackers reveals levels of daily activity and rest but recognizing the behavior of the dog would provide additional information, especially when the dog is not under supervision. In this study, a performance of a 3D accelerometer movement sensor placed on the dog collar was evaluated in classifying seven activities during semi-controlled test situation with 24 dogs. Various features were extracted from the acceleration time series signals. The performance of two classifiers was evaluated with two feature scenarios: using all computed features and the ones given by forward selection algorithm. The highest overall classification accuracy for the seven behaviors was $76 \%$. The results are promising pro improving classification of specific behaviors by relatively simple algorithms.
\end{abstract}

\section{Author Keywords}

Canine; accelerometer; activity monitoring.

\section{ACM Classification Keywords}

H.5.m. Information interfaces and presentation: Miscellaneous

\section{INTRODUCTION}

Accelerometers are increasingly used in animal research for various purposes, such as estimating energy expenditure and assessing behaviors in wildlife studies $[6,14]$, and veterinary medical research $[9,11]$. In addition, the consumer-targeted accelerometer based activity trackers for dogs have become popular, Whistle and PetPace for example [21, 22]. Typically, these devices come along with a smartphone

Permission to make digital or hard copies of part or all of this work for personal or classroom use is granted without fee provided that copies are not made or distributed for profit or commercial advantage and that copies bear this notice and the full citation on the first page. Copyrights for thirdparty components of this work must be honored. For all other uses, contact the Owner/Author.

ACI18, December 4-6, 2018, Atlanta, GA, USA (C) 2018 Copyright is held by the owner/author(s). ACM ISBN 978-1-4503-6219-1/18/12.

DOI: https://doi.org/10.1145/3295598.3295602 application that can visualize activity data as daily total activity, mean activity classes, and how many hours dog has moved or rested during the day [18] There are few validated devices for canine activity monitoring such as ActiGraph GT3X / GT3X+ [19, 13] and Actical [8, 15]. However, these devices are primarily targeted for data logging (recording only raw accelerometer data); thus, providing accuracy for research purposes, but they do not classify behavior or motions. Classifying motions and body positions reliably, would be promising tools for efficiently recording and analyzing even more subtle dog behaviors. Improved detection of dog's everyday behaviors would be important, for example, for understanding particular behaviors when the dog is alone at home or in a pen. Monitoring dog's behavior would be important especially in the case of behavior problems, such as separation anxiety. Extracting behaviors from accelerometer data has also potential to provide an index of wellbeing and health status of the animal, for example by detecting stress and pain-related behaviors [12, $1]$.

Fine-grained classification of dogs' behaviors would benefit tracking for several purposes like monitoring while at home or pen or in case of problematic behaviors. The classification algorithms should be simple and reliable, based on data collected in relatively controlled test situations. The high sensitivity of accelerometers makes their placement and attachment critical for obtaining reliable and repeatable measurements. According to Hansen et al. the best placement for an activity meter, regarding the accuracy and practicality, appears to be ventral attachment to the neck collar [8]. However, Preston et al. state that accelerometers mounted tightly on back detected some differences between behaviors that devices fastened loosely, or attached to the neck collar could not detect [17].

Also few other studies have been published about classifying dog behaviors using movement sensors attached on the collar or on the dog harness. Ladha et al. achieved $68.6 \%$ accuracy using KNN classification for differentiating 16 behaviors in naturalistic environments and collar mounted accelerometer [10]. Den Uijl et al. reported that dynamic behaviors (i.e. walk, trot, canter/gallop, eat, drink, and headshake) could be correctly classified with $95 \%$ accuracy and static behaviors 
(i.e. sleep, inactive) with over $90 \%$ accuracy [18]. They used accelerometers in the collar and hierarchical one vs. the rest classification and segments of $\geq 2$ seconds for eating and drinking, and $\geq 4$ second segments for other behaviors. Gerencsér et al. used SVM to classify seven activities such as sitting and trotting with an accuracy of more than $80 \%$ using a 3 -axis accelerometer and a 3 -axis gyroscope attached on a harness at the back of 24 adult dogs [4]. In addition, even behaviors without significant body motion such as scratching and eating, have been successfully classified by an accelerometer [8].

The aim of the present study was to evaluate classification algorithms and feature selection with accelerometer data collected during seven different dog behaviors: standing, sitting, lying chest, walking, trotting, galloping, and sniffing. The data set was recorded from a wide range of dog breeds using a 3D accelerometer attached in the collar. The main extensions to the related work include (not all apply to every study): the use of simple and robust parametric classifiers, linear and quadratic discriminant analysis (LDA, QDA), application of forward feature selection, the use of twosecond data segments, allowing detection of short periods of behavior, and classifying all seven behaviors simultaneously with a single classifier.

\section{TEST SETUP}

To develop activity classification algorithms, we need accelerometer recordings where the activity of the dog at a given point of time can be verified from an independent source. Such datasets were not publicly available. Therefore, we had to record a new set of data as follows.

The data were collected at the University of Helsinki. The procedures were approved by the Ethical Committee for the Use of Animals in experiments at the University of Helsinki (minutes 5/2017). All dog owners signed an informed consent before participating in the study.

Data from 24 healthy pet dogs from 20 breeds were included in the analysis. The average age of the dogs was 5.5 years (range 1.7-9.7 years) and weight $24.1 \mathrm{~kg}$ (range 14-41 kg) (Table 1). Participants were recruited from the internet, for example using Facebook and similar social media applications.

\begin{tabular}{c|ccc} 
Breed & N & Weight & Age \\
\hline Australian Kelpie & 1 & 18 & 1.7 \\
Beauceron & 1 & 28 & 4.3 \\
Belgian Shepherd & 1 & 29 & 6.3 \\
Belgian Shepherd Groenendael & 1 & 20 & 7.1 \\
Border Collie & 2 & 17.5 & 4.3 \\
Bouvier & 1 & 30 & 2.8 \\
Bouvier des Ardennes & 1 & 22 & 4.7 \\
Bull Terrier (Miniature) & 1 & 17 & 2.6 \\
Crossbreed & 3 & 17.3 & 5.4
\end{tabular}

\begin{tabular}{c|ccc} 
Breed & N & Weight & Age \\
\hline English Springer Spaniel & 1 & 25 & 5.6 \\
Finnish Lapphund & 1 & 26 & 7.3 \\
Flat-Coated Retriever & 1 & 28 & 2.8 \\
German Shepherd & 2 & 33.5 & 7.4 \\
Golden Retriever & 1 & 28 & 3.7 \\
Hovawart & 1 & 41 & 2.8 \\
Labrador Retriever & 1 & 23 & 9.4 \\
Lagotto Romagnolo & 1 & 14 & 9.7 \\
Lapponian Herder & 1 & 22 & 8.4 \\
Smooth Collie & 1 & 18 & 8.2 \\
Vizsla & 1 & 25 & 5.1
\end{tabular}

Table 1. The breed, sample size $(\mathrm{N})$, average age in years and average weight in kilograms of the dogs participating in the study.

\section{Procedure}

The tests were performed in a dog sporting hall in a testing arena $(10 \mathrm{~m} \times 18 \mathrm{~m})$ covered with artificial turf. The test consisted of seven tasks: sit, stand, lie down, trot, walk, play and treat-search. Each task lasted three minutes. Except for play and treat-search, the tasks were the same as the behaviors we wanted to be able to classify. Dogs performed tasks sequentially, alternating static tasks (the first three) and motion behavior tasks (the other ones). Treat search was always performed as the final task of the sequence. In treat search task, small pieces of dry dog food were placed on the floor (spread on area of $4 \mathrm{~m} \mathrm{x} 4 \mathrm{~m}$ ) and dogs were allowed to search them by sniffing. Playing was performed in freestyle promoting intense activity, such as running after a ball, playing tug-of-war game or carrying out different tricks. Owners were allowed to give food rewards and command their dogs through the entire test. Dogs were on a leash and led by their owner or experimenter. The leash $(1.50 \mathrm{~m})$ was connected to a separate collar from the one where the sensor unit was attached. The collar with the sensor unit was placed closer to the dogs' head.

Three tri-axial accelerometer device, ActiGraph GT9X Link (ActiGraph LLC, Florida, USA) was taped tightly on ventral side of neck collar. The accelerometer data was sampled at $100 \mathrm{~Hz}$.

\section{BEHAVIORAL ANNOTATION}

The test sessions were video recorded with Panasonic HDCSD600 and Sony HDR-CX450 cameras positioned on opposite lateral walls and aimed towards the testing arena. The behavior of each dog was manually annotated from the video recordings post-hoc using the Observer XT 10.5 (Noldus, The Netherlands) software. One main behavior (standing, sitting, lying chest, walking, trotting, galloping, sniffing) at a time was coded and only behaviors of more than one second were included in the study. Static positions (standing, sitting, lying chest) were annotated when limbs did not move and there was no physical contact between handler and dog, except if a treat was given. The gait pattern 
in dynamic behaviors (walking, trotting and galloping) was included, if the pattern was clear and continuous without the dog leaning towards handler or pulling the leash thus affecting the gait pattern or body position. Galloping occurred mostly during the play task and sniffing during the treat-search (see Table 2 for Ethogram).

\begin{tabular}{|c|c|}
\hline Behavior & Description \\
\hline Standing & $\begin{array}{l}\text { The dog has the four extremities on the } \\
\text { ground, without the dog's torso touching } \\
\text { the ground. }\end{array}$ \\
\hline Sitting & $\begin{array}{l}\text { The dog has four extremities and the rump } \\
\text { on the ground. The dog can change balance } \\
\text { point from central to hip or vice versa. }\end{array}$ \\
\hline $\begin{array}{l}\text { Lying } \\
\text { chest }\end{array}$ & $\begin{array}{c}\text { The dog torso is touching the ground and } \\
\text { hips are in the same level as shoulders. The } \\
\text { dog can change balance point without } \\
\text { limbs. }\end{array}$ \\
\hline Walking & $\begin{array}{l}\text { 4-beat gait where the dog moves its } \\
\text { extremities at a slow speed, legs are moved } \\
\text { one by one in the order: left hind leg, left } \\
\text { front leg, right hind leg, and right front leg. } \\
\text { The dog moves straight forward or at } \\
\text { maximum } 45 \text { degrees angle. }\end{array}$ \\
\hline Trotting & $\begin{array}{l}\text { 2-beat gait where the dog lifts and puts } \\
\text { down extremities in diagonal pairs at a } \\
\text { speed faster than walking. }\end{array}$ \\
\hline Galloping & $\begin{array}{l}\text { 3- or 4-beat gait where the dog lifts and } \\
\text { puts down both front and rear extremities in } \\
\text { a coordinated manner, in 1-2-3-beat gait } \\
\text { (canter) or in 1-2-3-4 beat gait (gallop). All } \\
\text { four extremities are in the air at some point } \\
\text { in every stride. }\end{array}$ \\
\hline Sniffing & $\begin{array}{l}\text { The dog has its head below its back line } \\
\text { and moves its muzzle close to the ground. } \\
\text { The dog walks stands or performs another } \\
\text { slow movement, but its chest and bottom } \\
\text { do not touch the ground. Taking food from } \\
\text { the ground and eating it can be included } \\
\text { (eating is not coded separately). }\end{array}$ \\
\hline
\end{tabular}

Table 2. Ethogram containing the behaviors included in the classification.

\section{ANALYSIS METHODS}

The raw time series data produced by the accelerometers were saved and analyzed offline. The data were segmented and various features were calculated for each segment. The features were labelled according to the annotations and used for classification of the behaviors.

\section{Feature extraction and labelling}

A wide variety of features have been used for classification of human activities in studies where accelerometers have been attached to wrist and thigh [16] or waist [5]. The time series signals recorded from the accelerometers were segmented into windows of 2 seconds. A total of 27 features were calculated for each segment:

- F1, Total activity: sum of standard deviation in all three axes.

- F2, Position offset: Euclidean distance from the robust mean obtained while the dogs were standing still.

- F3, Number of mean crossings, sum of $x, y$ and $z$ axes.

- F4 - F6, Mean value of each axis, x, y, z. Three features

- F7 - F27, Interpolated inverse empirical cumulative distribution function (ecdf). Seven values for each axis, total of 21 features

The ecdf features were calculated by shape-preserving piecewise cubic interpolation from the cumulative distribution function $\mathrm{P}_{\mathrm{c}}(\mathrm{x})=\mathrm{P}(\mathrm{X} \leq \mathrm{x})[7,10,3]$. For each of those points $\mathrm{p}_{\mathrm{i}}$ we estimated the value $\mathrm{X}_{\mathrm{i}}$ for which $\mathrm{P}\left(\mathrm{X} \leq \mathrm{X}_{\mathrm{i}}\right)$ $=p_{i}$. We used seven values of $p_{i}$, evenly distributed between 0 and 1 , in each $x, y$ and $z$ axis (21 features). Examples from normal distribution and one two-second window accelerometer at the neck, $\mathrm{x}$ direction are presented in Figure 1.
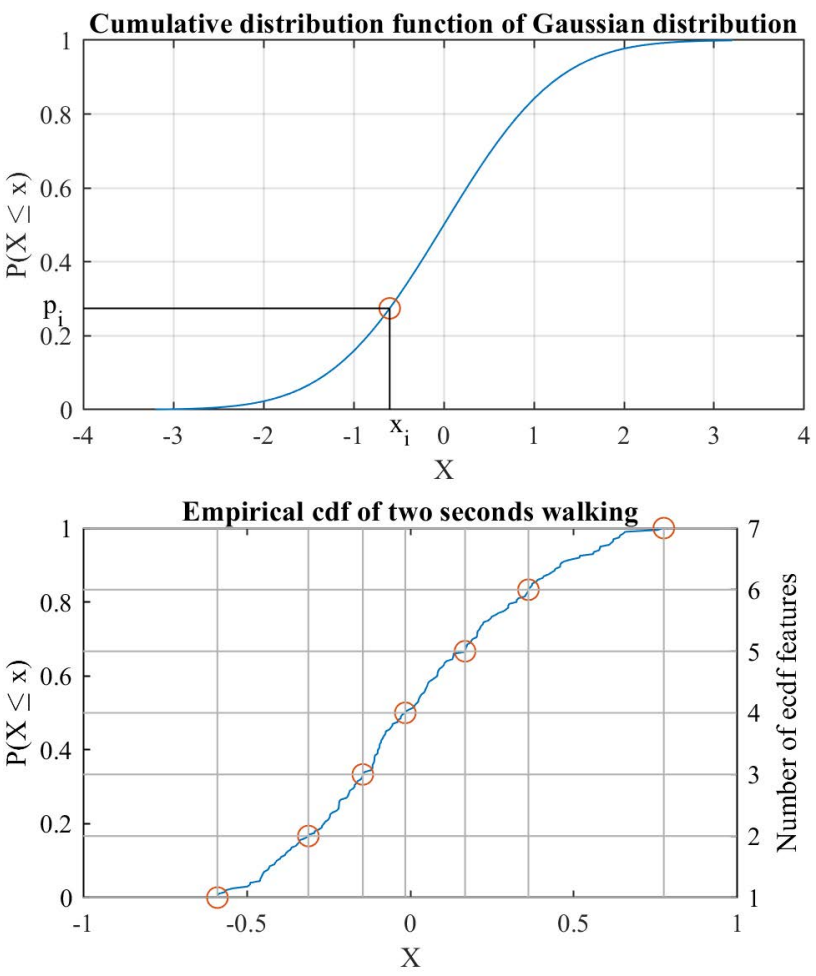

Figure 1. Examples of ecdf features. Top: one point from normal distribution at $P(X \leq x)=p_{i}$. Bottom: example of actual data evaluated at seven values of $p_{i}$ evenly spaced between 0 and 1.

The true classes of the segments were assigned according to the annotations from the videos. A behavior class was assigned to a segment if the annotated behavior was happening during a minimum of $75 \%$ of the segment. The 
tests from the 24 dogs provided 10367 instances of labelled data. Table 3 shows how the observations were distributed among the activities.

\begin{tabular}{c|c} 
Behavior & Number \\
\hline Standing & 1742 \\
Sitting & 2071 \\
Lying chest & 2033 \\
Walking & 1305 \\
Trotting & 1328 \\
Galloping & 158 \\
Sniffing & 1730
\end{tabular}

Table 3. The number of observations in each behavior class.

\section{Classification and feature selection}

The performance of two classifiers were evaluated in this study: linear and quadratic discriminant analysis classifier (LDA and QDA). The classification results were calculated using two feature sets: using all the 27 features listed in the previous section, and using features selected by a forward feature selection method, separately for both classifiers.

The forward selection procedure selects a subset of features that provides the best classification result. It includes features that best classify the data, adding them sequentially one by one, until there is no improvement in classification accuracy.
All classifications, including the forward selection, were calculated using 10-fold cross validation, where the data set was randomly divided into 10 groups. Each group at a time was left as the test set and the classifier was identified using the rest of the data. The final results are the averages of the predictions of the test groups in the ten repetitions.

\section{RESULTS}

The correct classification results for the two classifiers and the two feature sets are presented in Table 4 . All results were calculated using the same 10-fold cross validation partitioning.

\begin{tabular}{c|cc} 
Features & LDA & QDA \\
\hline All 27 & $73.9 \%$ & $73.4 \%$ \\
Selected & $72.7 \%(8)$ & $76.0 \%(5)$
\end{tabular}

Table 4. Classification accuracies. The number of the features included by the forward selection is shown in the parenthesis.

Total activity (F1) was the only feature included in both classifiers. The remaining features for LDA were the position offset and the number of mean crossings (F2 and F3) and five of the ecdf features; numbers 4, 5 and 6 for $\mathrm{X}$ axis and number 7 for both $Y$ and $Z$ axis (See Figure 1). The other features for QDA were the mean value of $X$ axis (F4) and the ecdf features number 7 of $\mathrm{X}$ axis, 6 of $\mathrm{Y}$ axis and 3 of $\mathrm{Z}$ axis.

The overall accuracies using all features were similar. However, with the selected features QDA provided slightly better accuracy and used fewer features. Confusion matrices in Figure 2 provide a detailed view of the classification

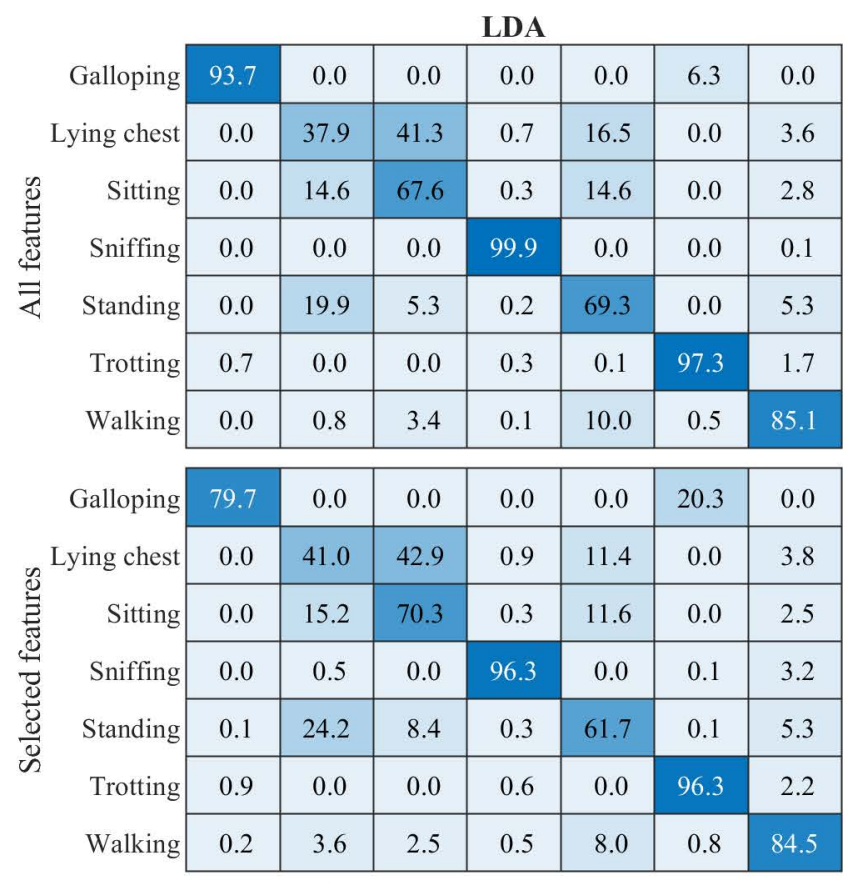

\begin{tabular}{|c|c|c|c|c|c|c|}
\hline 98.1 & 0.0 & 0.0 & 0.0 & 0.0 & 1.9 & 0.0 \\
\hline 0.0 & 33.1 & 35.7 & 1.1 & 21.9 & 2.7 & 5.5 \\
\hline 0.1 & 6.4 & 64.7 & 0.2 & 20.1 & 2.1 & 6.4 \\
\hline 0.6 & 0.0 & 0.0 & 99.3 & 0.0 & 0.1 & 0.0 \\
\hline 0.1 & 6.0 & 7.4 & 0.4 & 77.2 & 1.0 & 7.9 \\
\hline 2.2 & 0.0 & 0.0 & 0.2 & 0.0 & 97.2 & 0.4 \\
\hline 0.5 & 6.1 & 3.8 & 0.3 & 3.9 & 2.0 & 83.4 \\
\hline 74.7 & 0.0 & 0.0 & 0.0 & 0.0 & 25.3 & 0.0 \\
\hline 0.0 & 39.1 & 42.7 & 2.2 & 10.8 & 0.3 & 4.9 \\
\hline 0.0 & 9.6 & 72.8 & 0.6 & 12.7 & 0.1 & 4.2 \\
\hline 0.2 & 0.0 & 0.0 & 99.7 & 0.0 & 0.0 & 0.1 \\
\hline 0.1 & 6.3 & 9.0 & 0.8 & 80.1 & 0.2 & 3.6 \\
\hline 1.0 & 0.0 & 0.0 & 0.4 & 0.0 & 98.1 & 0.5 \\
\hline 0.2 & 9.0 & 3.8 & 1.0 & 5.5 & 1.0 & 79.6 \\
\hline
\end{tabular}

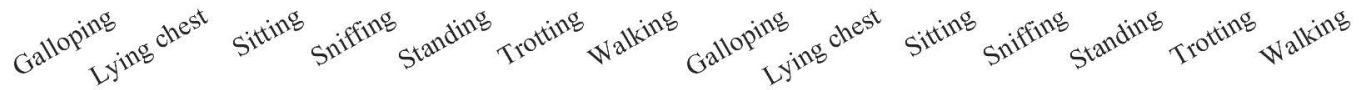

Figure 2 Confusion matrices of both classifiers and feature sets. 
performance for each behavior. The most challenging behaviors to classify were lying down, sitting, and standing. Correct classification rates for Lying down segments varied between $33 \%$ and $42 \%$. They were often misclassified as Sitting or Standing (for example, $35.7 \%$ and $21.9 \%$, respectively by QDA and all features).

The classification results were calculated also separately for each dog to study the differences between individuals. The distributions of the results for the QDA classifier are shown in Figure 3.

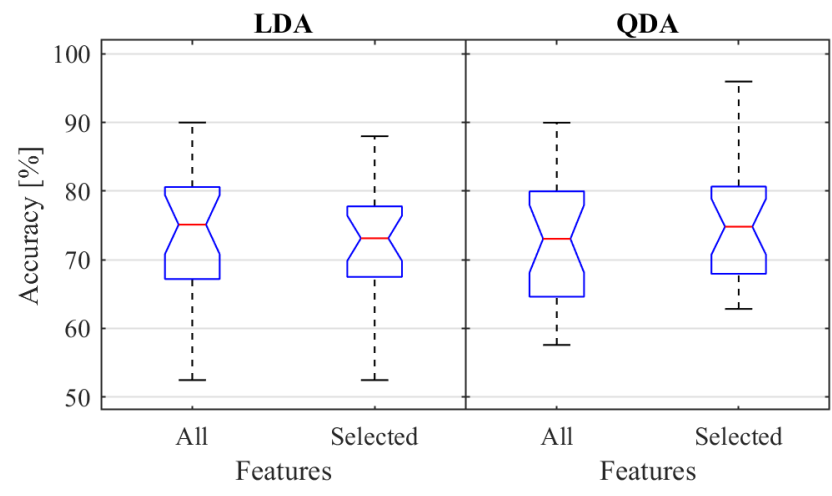

Figure 3. Boxplots of the classification accuracy distributions between the 24 dogs.

The medians of the four cases were not significantly different as the notched areas in the middle of the boxes overlap. The accuracies of LDA were between $52 \%$ and $90 \%$. Even though the median did not differ, the range of correct classifications for individual dogs achieved with QDA remained at higher levels than with LDA. The lowest value provided by QDA using all features was 58\% and selected features provided a range between $63 \%$ - 96\%.

\section{DISCUSSION}

There were no significant differences in the performance of the two classifiers. The feature selection improved the performance of QDA. While the operation principle of the LDA and QDA classifiers is basically the same, and they differ only by the identification of covariance matrices this result can be considered not surprising. The quadratic class boundaries in the more complicated QDA classifier did not provide significant improvement in cross-validated results with this particular data set.

While the initial number of features (27) was not particularly high, it was also reasonable that using feature selection did not improve the classification accuracy significantly. However, simple models, such as classifiers, using fewer features, were less prone to overfitting and therefore generalized better for predicting new data.

One challenge in this study was that the second collar used for the leash had a possibility to contact the sensor collar and, thus, disturb the signal. Further, it was seen from the data (also reported by the researchers performing the data collection) that the sensor did not always stay in the intended orientation, which obviously caused problems, especially in detection of the static behaviors.

Medium to large sized dog breeds participated in this study. The functionality of the classifiers should be tested with a larger dog population including smaller as well as dogs with deviant body structure (e.g. short legs). Ladha et al. [10] found better global accuracy for differentiating behaviors in small and medium sized dogs than in large ones.

\section{CONCLUSIONS AND FUTURE WORK}

The results are promising pro improving classification of specific behaviors by relatively simple algorithms. The highest overall classification accuracy for the seven behaviors was $83 \%$. On the other hand, the steady behaviors, sitting, standing and lying down, in particular, were hard to differentiate with an accelerometer attached on the collar. The challenge in such detailed classification for real life applications relates to the positioning of the accelerometer. Either the device should always be positioned at exactly the same orientation or the orientation should be recalibrated for the feature calculation after each time the sensor is attached or when its orientation has potentially changed.

Our future work includes evaluating a wider range of classifiers and using more features from the gyroscope data, and additional feature selection methods. Another direction for future works is to utilize multiple time resolution approach for fast and slow events, and classification in multiple stages to subgroups of behaviors. For real life applications, the complexity of the classification, including the feature extraction, needs to be considered. Doing all the computation in an embedded device requires more computing power and therefore increases the battery usage. However, this would reduce the amount of the data to transfer from the sensor.

\section{REFERENCES}

1. Dorothy C. Brown, Raymond C. Boston, John T. Farrar. 2010. Use of an activity monitor to detect response to treatment in dogs with osteoarthritis. $\mathrm{J} \mathrm{Am}$ Vet Med Assoc, 237, 1: 66-70.

2. Nancy Clarke, David Fraser. 2016. Automated monitoring of resting in dogs. Appl Anim Behav Sci 174: 99-102.

3. David R. Cox and David Oakes. 1984. Analysis of Survival Data. Chapman \& Hall.

4. Linda Gerencsér Gábor Vásárhelyi, Máté Nagy Tamas Vicsek, Adam Miklósi . 2013. Identification of behaviour in freely moving dogs (Canis familiaris) using inertial sensors. PLoS One 8, 10:1-14.

5. Piyush Gupta and Tim Dallas. 2014. Feature Selection and Activity Recognition System Using a Single Triaxial Accelerometer. IEEE Transactions on Biomedical Engineering 61, 6: 1780-1786

6. Lewis G.Halsey, Emily L.C.Shepard, Rory P.Wilson. 2011. Assessing the development and application of 
the accelerometry technique for estimating energy expenditure. Comparative Biochemistry and Physiology, Part A 158 ,3: 305-314.

7. Nils Y. Hammerla, Reuben Kirkham, Peter Andras, Thomas Plotz. . 2013. On Preserving Statistical Characteristics of Accelerometry Data using their Empirical Cumulative Distribution. In Proceedings of the 2013 International Symposium on Wearable Computers (ISWC '13), 65-68.

8. Bernard D. Hansen, Duncan X. Lascelles, Bruce W. Keene, Allison K. Adams, Andrea E. Thomson. 2007. Evaluation of an accelerometer for at-home monitoring of spontaneous activity in dogs. Am J Vet Res 68, 5: 468-475.

9. J. Helm, A. McBrearty, S. Fontaine, R. Morrison, P. Yam. 2016. Use of accelerometry to investigate physical activity in dogs receiving chemotherapy. Journal of Small Animal Practice 57, 11: 600-609.

10. Cassim Ladha, Nils Hammerla, Emma Hughes, Patrick Olivier, Thomas Plotz. 2013. Dog's Life: Wearable Activity Recognition for Dogs. In ACM International Joint Conference on Pervasive and Ubiquitous Computing (UbiComp '13), 415-418.

11. Dianne Little, Stephen Johnson, Jonathan Hash, Steven A. Olson, Bradley T. Estes, Franklin T. Moutos, B. Duncan X. Lascelles, Farshid Guilak. 2016. Functional outcome measures in a surgical model of hip osteoarthritis in dogs. Journal of Experimental Orthopaedics 3, 17: 1-16.

12. R. Morrison, J. J. Reilly, V. Penpraze, E. Pendlebury, P. S. Yam. 2014. A 6- month observational study of changes in objectively measured physical activity during weight loss in dogs. Journal of Small Animal Practice 55, 11: 566-570.

13. Ryan Morrison, Victoria Penpraze, Ruth Greening, Tom Underwood, John J. Reilly, Philippa S. Yam. 2014 Correlates of objectively measured physical activity in dogs. Vet J. 199, 2: 263-267.

14. Ran Nathan, Orr Spiegel, Scott Fortmann-Roe, Roi Harel, Martin Wikelski, Wayne M. Getz. 2012. Using tri-axial acceleration data to identify behavioral modes of free-ranging animals: general concepts and tools illustrated for griffon vultures. J. Exp. Biol 215, 986996.

15. Anastasia M. Olsen, Richard B. Evans, Felix M. Duerr. 2016. Evaluation of accelerometer inter-device variability and collar placement in dogs. Vet Evid 1, 2: $1-7$.

16. Susanna Pirttikangas, Kaori Fujinami and Tatsuo Nakajima. 2006. Feature Selection and Activity Recognition from Wearable Sensors. In Ubiquitous Computing Systems (UCS 2006); 516-527.
17. Timothy Preston, Wendy Baltzer, Stewart Trost. 2012. Accelerometer validity and placement for detection of changes in physical activity in dogs under controlled conditions on a treadmill. Res Vet Sci 93, 1: 412-416.

18. Ingrid den Uijl, Constanza B. Gómez Álvarez, David Bartram, Yoni Dror, Robert Holland, Alasdair Cook . 2017. External validation of a collar-mounted triaxial accelerometer for second-by-second monitoring of eight behavioural states in dogs. PLoS ONE 12, 11: 113.

19. P. S. Yam, V. Penpraze, D. Young, M. S. Todd, A. D. Cloney, K. A. Houston-Callaghan, J. J. Reilly. 2011. Validity, practical utility and reliability of ActiGraph accelerometry for the measurement of habitual physical activity in dogs. J Small Anim Pract 52, 2: 86-91.

20. POIS!!!Masanori Yamada, Mikihiko, Tokuriki. 2000. Spontaneous activities measured continuously by an accelerometer in beagle dogs housed in a cage. $J$ Vet Med Sci 62, 4: 443-447.

21. Jonathan M. Yashari, Colleen G. Duncan and Felix M. Duerr. 2015. Evaluation of a novel canine activity monitor for at-home physical activity analysis. $B M C$ Veterinary Research 11:146.

22. B. Belda, M. Enomoto, B.C. Case, B.D.X. Lascelles. 2018. Initial evaluation of PetPace. Vet J 237: 63-68. 